\title{
EDITORIAL
}

\section{Practical usefulness of heart rate monitoring in physical exercise}

\section{Utilidad práctica de la monitorización de la frecuencia cardiaca durante el ejercicio físico}

\author{
Camilo E. Povea ${ }^{\mathrm{a}, \mathrm{b}, *}$, Arturo Cabrera ${ }^{\mathrm{c}}$ \\ ${ }^{a}$ Cardiovascular Prevention and Sports Medicine Unit, Fundación Cardioinfantil Instituto de Cardiología- Colombia \\ ${ }^{\mathrm{b}}$ Department of Internal Medicine, Sports Medicine Postgraduate Degree, Universidad Nacional de Colombia \\ ' Escuela Nacional del Deporte [National School of Sports], Cali, Colombia
}

Received 24 May 2018; accepted 25 May 2018

Available online 1 June 2018

Engaging in directed, programmed physical activity, or even promoting a more active lifestyle, is increasingly widespread. It is clear that the benefits of regularly engaging in these activities constitute a protective factor against the development of chronic diseases. ${ }^{1}$ These favorable effects can be seen in primary and secondary prevention in patients with heart failure, coronary disease or arterial hypertension, ${ }^{2}$ and, more broadly, in any sedentary individual, with or without cardiovascular risk factors. In light of this, the number of people who may benefit from a personalized physical activity program is constantly growing, leading to a more frequent use of methods that streamline and facilitate the development of these programs. It is indispensable for healthcare professionals, and particularly physicians, to be able to provide recommendations regarding the best way to quantify the intensity of a given physical effort, as well as the proper use and measurement of heart rate (HR) as an indicator of this effort. Manual HR measurement may produce inaccurate results ${ }^{3}$ and even at times require inter-

DOI of original article:

https://doi.org/10.1016/j.rccar.2018.05.001

* Corresponding author.

E-mail address: cpovea@cardioinfantil.org (C.E. Povea). ruption of the exercise in order to employ it correctly, thus altering the actual result. The use of an electrocardiogram and/or Holter is impossible on a regular basis, and its usefulness in the field is practically nil.

The methodology for monitoring physical or sports training (classified as resistance activities) has progressed significantly. ${ }^{4}$ From an almost exclusive use of subjective perception of effort we have progressed to a much more individualized, adjusted, streamlined, and to a certain degree, much more effective level of training planning. The use of devices which simultaneously read multiple variables, both internal (HR, respiratory rate, temperature) as well as external (accelerometry, speed, height, temperature, etc. $)^{5}$ has permitted specific monitoring of the intensity and even the duration of physical activity. . $^{4} 6$

Scientific monitoring of training sessions has led to a considerable advance in the physiological measurement of humansádaptation to physical effort. The massification of the use of devices and applications which monitor HR constantly during exercise or even during competition ${ }^{7}$ makes it easier to include people in sporting events geared towards the general public (10 K races, bicycle tours, hikes, etc.). In the field of sports sciences and clinically applied sports medicine, the emergence of numerous and modern individual monitoring devices is increasingly common. Generally, 
we can speak of broad groups of sensors: movement sensors (pedometers, accelerometers, and GPS) and physiological sensors (heart rate monitors, temperature sensors and integrated sensors). ${ }^{5}$ Overall, many of these contribute to improving the confidence of those who engage in exercise, and provide a certain degree of security on a cardiovascular level. However, during physical exertion, individuals may occasionally register HRs which tend to be abnormally elevated. It is important to remember that although heart rate monitors were designed for healthy athletes with a baseline sinus rhythm, these devices are capable of detecting exercise-induced arrhythmias. However, they do not detect QRS complex morphology, let alone atrial signs ${ }^{8}$, and should therefore be used cautiously. Although many healthcare professionals may broadly recognize the relevance of continuous ongoing $\mathrm{HR}$ monitoring during exercise, heart rate rhythm monitors continue to be very frequently misused or even discontinued after a time by users and patients when their functions, even the most basic ones, are not understood.

\section{Heart rate as an indicator of the bodyś level of exertion}

In physical exercise, the relationship between muscular strength or running exercise intensity is generally linear. For any increase in strength and/or intensity there is a corresponding increase in HR. However, these increases may also be related to the stress associated with the activity, and not necessarily to the increased work load. This may be seen most easily in areas in which exercise is not very intense (close to rest), where increases of up to 40 beats per minute may be found, without an increased energy requirement.

With intense physical exercise and an increased work load, the lack of a rise in heart rate is considered to be a plateau, which, in many cases, is defined as the maximum HR.

\section{Maximum HR}

The HRmax achieved by each individual is completely personal and influenced by the central nervous system (but there are also environmental conditions which may influence it (temperature, humidity, altitude, etc.)). ${ }^{10}$ Thus, the relationship between HR and oxygen consumption is purely individual. ${ }^{10,11}$ Maximum HR decreases with age, possibly due to modifications in the autonomic response, altered sensitivity of the cardiomyocytes to catecholamines and/or the reduction of their reuptake, and a change in baroreflex sensitivity. ${ }^{14}$

The indiscriminate use of the 220 - age equation for calculating HRmax overestimates the HR figure in young people and underestimates it in older individuals. ${ }^{12} \mathrm{~A}$ longitudinal study shows a smaller reduction in HRmax with age $(0.7$ instead of 1 beat per year) and a lower $y$-intersection point regardless of the level of training or sex. ${ }^{11}$ Apparently, the HRmax value may vary according to the sport practiced, ${ }^{10}$ or the level of training. For equivalent strength exertions (running vs bicycle exertion) in the same individual under similar environmental conditions, the HR reached during running may be around 10 beats per minute higher than in bicycling. ${ }^{10}$ This also suggests different formulas for calculating maximum effort depending on the sport: (165 - $0.5 \mathrm{x}$ age (years) \pm 5 beats for running; $160-0.5 \times$ age (years) \pm 5 beats for cycling). If sports in which the body position can modify venous return are considered, such as swimming, maximum heart rates 5 to 10 beats lower may be observed. ${ }^{13}$

\section{Cardiovascular drift}

During long-term exertion: in an exercise lasting more than 20 minutes with a constant intensity, an HR drift is seen, consisting in an increased HR. ${ }^{9}$ The drift is caused by a progressive reduction in systolic ejection volume secondary to dehydration, especially with elevated environmental temperatures, which leads to a decreased effective volemia $(100-120 \mathrm{ml})$. This must be compensated for by an accelerated $\mathrm{HR}$ in order to guarantee the same $\mathrm{O}_{2}$ supply to the involved muscles. Thus, if an increased HR is observed during the training session despite a constant intensity, rehydration should be encouraged to avoid cardiovascular drift.

\section{Heart rate zones}

Most software programs or applications which currently accompany HR devices and are used for personalized training recognize and use five heart rate zones, classified from 1 to $5^{9}$. The most valid method for establishing training zones with the HR is a laboratory lactate threshold test on a treadmill or cycle ergometer which involves a staged increase in work load and blood sampling at the end of each stage. These zones may be HR intervals of 5 to 10 beats per minute for each individual. The assessment may also be carried out using ergospirometry which is necessary in both subjects with poor physical capacity due to pulmonary or cardiovascular disease as well as in high performance athletes. The maximum peak is determined in this way, as well as the first aerobic or resistance threshold (ventilatory adaptation), and the second anaerobic or resistance threshold. However, the interpretation of the thresholds is, in many cases, operator-dependent.

The maximum predicted HR by age is a value which, in general, is not in line with reality. The best way of establishing it is through a laboratory or field test adapted for the individual who requires it. A field test may be useful for asymptomatic, healthy subjects without risk factors or family history.

\section{Resting HR}

It is not absolutely necessary to establish an initial resting $\mathrm{HR}$ in order to carry out the calculations; many factors may condition it, and it may vary from one day to the next. To correctly ascertain this HR, it should be done in the morning before getting out of bed, or immediately after waking, preferably lying supine, in a fasting state, for at least five minutes for three consecutive days, and repeated frequently. While this may be a good indicator of physical fitness, or in cases where it is altered may reveal a physical or emotional overload, it does not definitely predict how much it changes with physical exertion. 


\section{Recovery HR}

Tracking this variable may be very interesting as long as the effort the individual undergoes is accurately established. Recovery HR depends on the fitness level and is not very sensitive to external factors. ${ }^{17}$

In practical terms, an individual is considered to have a good recovery capacity after exertion if his/her HR drops by at least a third compared to the maximum achieved during exercise, by the third minute of passive recovery. ${ }^{18}$

\section{Heart rate reserve}

Heart rate reserve is widely used to establish the zones and corresponds to the difference between maximum HR and resting HR. Clearly, the higher it is, the more relevant it becomes as an indicator of exertion, and the more important it will be in determining the role of $\mathrm{HR}$ in the progression of oxygen consumption $\left(\dot{V} \mathrm{O}_{2}\right)$ on exertion.

\section{Work zones}

The Karvonen formula establishes a frequency goal which is equal to resting HR plus a percentage of the HR reserve. ${ }^{15} \mathrm{HR}$ Reserve $=$ (resting HR - maximum HR). To calculate the training HR, we take $85 \%$ of the previously calculated HR reserve and add the resting HR. While this method is very accurate it is conditioned by the resting HR variations previously described. The simplest method is the one that classically uses the percentage (\%) of maximum $\mathrm{HR}$ and the \% of $\dot{\mathrm{V}} \mathrm{O}_{2}$ which remains almost identical in any individual performing a session at $85 \%$ of his/her $\dot{V} O_{2 \max }$, which more or less corresponds to requesting $90-95 \%$ of his/her HR max.

\section{Training zones}

Zone 1 The HR values are used especially for recovery processes and to work on fatigue resistance and efficiency. The values correspond to 50 to $60 \%$ of the maximum HR.

Zone 2 for resistance training. This zones intensity produces cardiovascular improvements with training but does not require more time. The values correspond to the range of 60 to $70 \%$ of the maximum $\mathrm{HR}$.

Zone 3 has a higher intensity, used for greater resistance training. This is the zone commonly used for speed training. The amount of zone 3 training should be limited (especially running), due to the pressure placed on the body with this training intensity. The values correspond to a range between 70 and $80 \%$ of the maximum heart rate.

Zone 4 The intensity is very close to the athletes lactate threshold. Work in this zone is considered intense, both physically as well as psychologically, and should generally be used at least once a week during a planned training session. However, it must be monitored and limited since the probability of musculoskeletal injuries may be higher. The values correspond to the range of 80 to $90 \%$ of the maximum HR.

Zone 5 is used for $\dot{V} O_{2 \max }$ training and must be used in moderation due to the extreme overload to which it can lead. The $\dot{V} \mathrm{O}_{2 \text { max }}$ is genetically determined and once the maximum limit of $\dot{\mathrm{V}} \mathrm{O}_{2 \text { max }}$ is achieved, it cannot be significantly increased. Training time should be used in other areas. The values correspond to a range of 90 to $100 \%$ of the maximum HR.

Another approach to training monitoring is quantification of the training load, which considers two key concepts: the internal load, which represents the physiological stress in response to the effort exerted during training sessions, on the one hand; and the external load corresponding to the external training variables such as duration, speed, acceleration, watts, etc., on the other hand. ${ }^{20}$

The following formula may be used to calculate the training load:

Training load = internal load $\mathrm{x}$ external load (measured in arbitrary units)

Heart rate is one of the easily quantifiable internal load variables, while the length of the session is a practical way of measuring external load. ${ }^{20}$

Traditionally, training monitoring focuses on only one of the two variables which make up the load, which results in a partial picture of the stimulus-response duality. Thus, integrating both the internal and external load in monitoring can facilitate decision making with regard to the work request for a given session, allowing a personalized monitoring of the training.

One way of individualizing training through load monitoring is the TRIMP (Training Impulse) method. This is a mathematical model which quantifies the total load of an exercise session ${ }^{20}$ integrating the internal load (HR) with the external load (length of session).

In order to calculate TRIMP, you need to know the resting heart rate, maximum heart rate (measured in a maximum effort), average heart rate during the exercise session and the length of this session. ${ }^{21}$

$\operatorname{TRIMP}=\mathrm{D} \times(\Delta F C)] \times \mathrm{e}^{(\mathrm{b} \times \Delta \mathrm{Fc})}$

Where $\triangle F C$ is equal to:

[(Average HR during the session - Resting HR)/(Maximum $H R$ - Resting $H R)]$,

the constant $e=2.718$; and $b$ reflects the exercises degree of stress, associated with the lactate curve during exertion, which behaves differently in men and women. For women, $b=1.67$, and for men, $b=1.92 .{ }^{21}$

This training load quantification model is applicable for both healthy people 22 as well as patients with heart disease. ${ }^{23}$ In the case of patients with chronic heart failure, a cardiac rehabilitation program is part of their treatment, with a level 1 evidence. ${ }^{24,25}$ However, the optimal stimulus in intensity and duration is controversial, showing a tendency in recent studies towards a greater benefit with exercise in intervals compared to continuous exercise . ${ }^{26}$ However, the stress generated for each method is not necessarily the same, which makes it difficult to compare them. lellamo et al. compared two exercise programs for 12 weeks in patients with heart failure; one with continuous exercise and the other with interval training. They used the TRIMP method to individually monitor the training loads, seeking to make the total load equal in both groups. They found an increased $\dot{V} \mathrm{O}_{2 \text { max }}$ and ventilatory threshold in both methods, without significant differences between the groups. 
This shows that individual monitoring of the training load allows a better interpretation of the requested stimulus and its possible adaptations. ${ }^{23}$

\section{Generalities of HR monitors}

Heart rate monitors are simple electronic devices made up of two elements, a transmitter (generally located on the chest) which captures the heartś electrical signal, corresponding to the R wave on the electrocardiogram, ${ }^{3}$ and sends it towards a clock or receptive device which converts the data into numbers, displaying and storing the figures. More recently, other systems have been included which detect movement and travel speed. With the miniaturization of devices, they have become more sophisticated, offering a multiplicity of functions. Some more recent models include transmitters on the wrist itself, although if you take into account that an individual may carry out different types of exercise, the precision of these latter devices is not greater than those having the transmitting electrodes on the chest. Recent studies show various degrees of correlation with electrocardiogram tracings depending on the exercise modality and/or the brand of equipment, with the most adequate transmitter being the one placed on the chest, which would be the most recommended for patients and athletes. ${ }^{19}$

\section{Basic functions}

Currently, the advances obtained in the transmitting electrode are not comparable to those of the receptors. The materials from which the supports which hold the electrodes are made have become flexible, and therefore more comfortable, and the receptors show good correlations (0.97) with the comparative reference values in stress tests (a 3-4 beat difference). ${ }^{19}$ Currently, some transmitters, using wireless technology, connect to intelligent phones through applications. ${ }^{16}$ Most of the proposed models are coded, which enables them to avoid interference with other nearby devices, and the selection of different wavelengths makes it hard to find two overlapping signals. The HR is generally displayed on the screens every 5 seconds, or every 15 seconds in the most basic models. Only some high-end devices provide beat to beat storage. ${ }^{6}$ The work zones can be memorized, and there are usually one or several audible alarms to indicate work in certain zones. Some devices subjectively calculate the physical fitness index and equate it with the calculation of maximum oxygen consumption. The precision of this method is highly debatable and its usefulness can be improved if periodic measurements can be taken, and in models which use a beat to beat recording of the HR, allowing an analysis of heart rhythm variability. 6,17

In conclusion, training monitoring should be carried out in an orderly, systematic fashion, with practical tools which show the training load. They should, as much as possible, monitor both the external load as well as the internal load, and it is in this latter point where heart rate plays an important role for individual planning and decision making, both in healthy people as well as in patients with heart disease.

\section{References}

1. https://aspe.hhs.gov/basic-report/physical-activityfundamental-preventing-disease.

2. Lavie CJ, Thomas RJ, Squires RW, Allison TG, Milani RV. Exercise training and cardiac rehabilitation in primary and secondary prevention of coronary heart disease. Mayo Clin Proc. 2009;84:373-83.

3. Laukkanen RM, Virtanen PK. Heart rate monitors: state of the art. J Sports Sci. 1998;16 Suppl:S3-7.

4. Wright SP, Hall Brown TS, Collier SR, Sandberg K. How consumer physical activity monitors could transform human physiology research. Am J Physiol Regul Integr Comp Physiol. 2017, http://dx.doi.org/10.1152/ajpregu.00349.2016, 312: R358-R367, First published January 42017.

5. Li RT, Kling SR, Salata MJ, Cupp SA, Sheehan J, Voos JE. Wearable Performance Devices in Sports Medicine. Sports Health. 2016 Jan-Feb;8:74-8, http://dx.doi.org/10.1177/1941738115616917. Epub 2015 Nov 11.

6. Achten J, Jeukendrup AE. Heart rate monitoring: applications and limitations. Sports Med.;. 2003;33:517-38, http://dx.doi.org/10.2165/00007256-200333070-00004. PubMed.

7. Cardinale M, Varley MC. Wearable Training-Monitoring Technology: Applications Challenges, and Opportunities. Int J Sports Physiol Perform. 2017 Apr;12 Suppl 2:S255-62, http://dx.doi.org/10.1123/ijspp.2016-0423. Epub 2016.

8. Gajda R, Biernacka EK, Drygas W. Are heart rate monitors valuable tools for diagnosing arrhythmias in endurance athletes? Scand J Med Sci Sports. 2018 Feb;28:496-516, http://dx.doi.org/10.1111/sms.12917. Epub 2017 Jun 16.

9. Gilman MB. The use of heart rate to monitor the intensity of endurance training. Sports Med. 1996 Feb;21:73-9.

10. Knoepfli-Lenzin C, Haenggli B, Boutellier U. Optimised heart rate formulae to monitor endurance training in sedentary individuals. J Sports Sci. 2014;32:557-62, http: / /dx.doi.org/10.1080/02640414.2013.843015.

11. Gellish RL, Goslin BR, Olson RE, McDonald A, Russi GD, Moudgil VK. Longitudinal modeling of the relationship between age and maximal heart rate. Med Sci Sports Exerc. 2007 May;39:822-9.

12. Tanaka H, Monahan KD, Seals DR. Age-predicted maximal heart rate revisited. J Am Coll Cardiol. 2001 Jan;37:153-6. PubMed PMID: 11153730.

13. Suk MH, Yu KH, Shin YA. Comparison of tests for measuring maximal exercise ability in elite swimmers. J Exerc Rehabil. 2016 Jun 30;12:209-15, http://dx.doi.org/10.12965/jer.1632606.303.

14. Kaye DM, Esler MD. Autonomic control of the aging heart. Neuromolecular Med. 2008;10:179-86, http://dx.doi.org/10.1007/s12017-008-8034-1. Epub 2008 Mar 13 Review PubMed PMID: 18338270.

15. Borresen J, Lambert MI. The quantification of training load, the training response and the effect on performance. Sports Med. 2009;39:779-95, http://dx.doi.org/10.2165/11317780-000000000-00000.

16. Bouts AM, Brackman L, Martin E, Subasic AM, Potkanowicz ES. The Accuracy and Validity of iOS-Based Heart Rate Apps During Moderate to High Intensity Exercise. Int J Exerc Sci. 2018 Jan 2;11:533-40, eCollection 2018.

17. Thomson RL, Bellenger CR, Howe PRC, Karavirta L, Buckley JD. Improved heart rate recovery despite reduced exercise performance following heavy training: A withinsubject analysis. J Sci Med Sport. 2016 Mar;19:255-9, http://dx.doi.org/10.1016/j.jsams.2015.02.010.

19. Gillinov S, Etiwy M, Wang R, Blackburn G, Phelan D, Gillinov $A M$, Houghtaling $P$, Javadikasgari $H$, Desai MY. Variable Accuracy of Wearable Heart Rate Monitors during Aerobic 
Exercise. Med Sci Sports Exerc. 2017 Aug;49:1697-703, http://dx.doi.org/10.1249/MSS. 0000000000001284.

20. McGuigan Mike. Monitoring training and performance in athletes. Human Kinetics. 2017.

21. Morton RH, Fitz-Clarke JR, Banister EW. Modeling human performance in running. J Appl Physiol (1985). 1990 Sep;69:1171-7.

22. Barrett S, Midgley A, Lovell R. PlayerLoad ${ }^{\mathrm{TM}}$ : reliability, convergent validity, and influence of unit position during treadmill running. Int J Sports Physiol Perform. 2014 Nov;9:945-52, http://dx.doi.org/10.1123/ijspp.2013-0418.

23. lellamo F, Manzi V, Caminiti G, Vitale C, Castagna C, Massaro M, Franchini A, Rosano G, Volterrani M. Matched dose interval and continuous exercise training induce similar cardiorespiratory and metabolic adaptations in patients with heart failure. Int J Cardiol. 2013 Sep 10;167:2561-5, http://dx.doi.org/10.1016/j.ijcard.2012.06.057.

24. Piepoli MF, Corrà U, Benzer W, Bjarnason-Wehrens B, Dendale P, Gaita D, McGee $H$, Mendes $M$, Niebauer J, Zwisler AD, Schmid JP, Cardiac Rehabilitation Section of the European Association of Cardiovascular Prevention and Rehabilitation. Secondary prevention through cardiac rehabilitation: from knowledge to implementation. A position paper from the Cardiac Rehabilitation Section of the European Association of Cardiovascular Prevention and Rehabilitation. Eur J Cardiovasc Prev Rehabil. 2010 Feb;17:1-17, http://dx.doi.org/10.1097/HJR.0b013e3283313592.

25. Piña IL, Apstein CS, Balady GJ, Belardinelli R, Chaitman BR, Duscha BD, Fletcher BJ, Fleg JL, Myers JN, Sullivan MJ. American Heart Association Committee on exercise, rehabilitation, and prevention. Exercise and heart failure: A statement from the American Heart Association Committee on exercise, rehabilitation, and prevention. Circulation. 2003 Mar 4;107:1210-25.

26. Wisløff U, Støylen A, Loennechen JP, Bruvold M, Rognmo $\varnothing$, Haram PM, Tjønna AE, Helgerud J, Slørdahl SA, Lee SJ, Videm V, Bye A, Smith GL, Najjar SM, Ellingsen Ø, Skjaerpe T. Superior cardiovascular effect of aerobic interval training versus moderate continuous training in heart failure patients: a randomized study. Circulation. 2007 Jun 19;115:3086-94. 